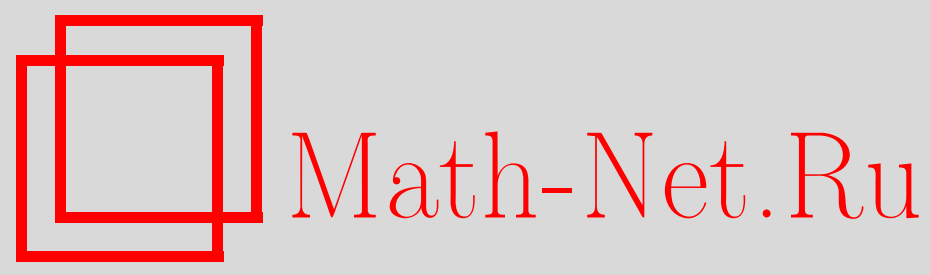

Ю. Н. Субботин, С. А. Теляковский, Нормы в $L$ периодических интерполяционных сплайнов с равноотстоящими узлами, Матем. заметки, 2003, том 74, выпуск 1, 108-117

DOI: https://doi.org/10.4213/mzm249

Использование Общероссийского математического портала Math-Net.Ru подразумевает, что вы прочитали и согласны с пользовательским соглашением http://www . mathnet.ru/rus/agreement

Параметры загрузки:

IP: 18.234 .197 .8

26 апреля 2023 г., $15: 29: 50$

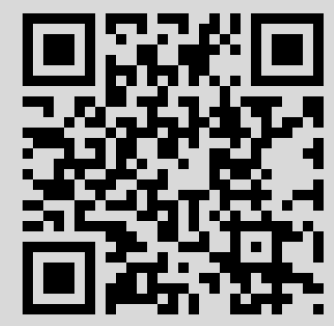




\title{
НОРМЫ В $L$ ПЕРИОДИЧЕСКИХ ИНТЕРПОЛЯЦИОННЫХ СПЛАЙНОВ С РАВНООТСТОЯЩИМИ УЗЛАМИ
}

\author{
Ю.Н. Субботин, С. А. Теляковский
}

Рассматривается множество $S_{r, n}$ периодических с периодом 1 сплайнов степени $r$ дефекта 1 с узлами в $n$ равноотстоящих точках $x_{i}=i / n$. Для наборов $\mathbf{y}=\left(y_{0}, y_{1}, \ldots\right.$, $\left.y_{n-1}\right)$ берутся сплайны $s_{r, n}(\mathbf{y}, x)$ из $S_{r, n}$, решающие интерполяционную задачу

$$
s r, n\left(\mathbf{y}, t_{i}\right)=y_{i}
$$

где $t_{i}=x_{i}$, если $r$ нечетно, и $t_{i}-$ середины отрезков [ $\left.x_{i}, x_{i+1}\right]$, если $r$ четно. Для норм $L_{r, n}^{*}$ оператора $\mathbf{y} \rightarrow s_{r, n}(\mathbf{y}, x)$ как оператора из $l^{1}$ в $L^{1}[0,1]$ установлена оценка

$$
L_{r, n}^{*}=\frac{4}{\pi^{2} n} \log \min (r, n)+O\left(\frac{1}{n}\right)
$$

с абсолютной постоянной в остаточном члене. Выяснена связь норм $L_{r, n}^{*}$ с нормами аналогичного оператора для непериодических сплайнов.

Библиография: 7 названий.

1. Введение. Пусть $S_{r, n}$-множество периодических с периодом 1 сплайнов степени $r$ дефекта 1 с узлами в $n$ равноотстоящих точках $x_{i}:=i / n, i=0,1, \ldots, n$.

В работе [1] была рассмотрена следующая задача.

Каждой непрерывной периодической с периодом 1 функции $f(x)$ ставится в соответствие сплайн $s_{r, n}(f, x)$ из $S_{r, n}$, интерполирующий функцию $f$ в точках

$$
t_{i}:=x_{i}+\frac{1+(-1)^{r}}{4 n}, \quad i=0,1, \ldots, n,
$$

т.е. в узлах $x_{i}$, если $r$ нечетно, и в серединах отрезков $\left[x_{i}, x_{i+1}\right]$, если $r$ четно. Заметим, что $t_{n}=1+t_{0}$. Существование и единственность интерполяционных сплайнов $s_{r, n}(f, x)$ известны, см. [2, с. 121].

В [1] изучалось асимптотическое поведение констант Лебега $L_{r, n}$ указанных интерполящионных сплайнов, т.е. норм оператора $f(x) \rightarrow s_{r, n}(f, x)$ как оператора из $C$ в $C$. Было доказано, что

$$
L_{r, n}=\frac{2}{\pi} \log \min (r, n)+O(1)
$$

Работапервого автора была выполненапри поддержке Российского фонда фундаментальных исследований, грант № 02-01-00764, и программы "Ведущие научные школы", грант № 00-15-96035, работа второго автора была выполнена при поддержке Российского фонда фундаментальных исследований, грант № 02-01-00787, программы "Ведущие научные школы", грант № 00-15-96047, и INTAS, грант № 99-01080. 
с абсолютной постоянной в остаточном члене.

Настоящая работа посвящена изучению аналогичного вопроса о нормах интерполяционных сплайнов из $S_{r, n}$ как операторов из $l^{1}$ в $L^{1}$.

Точная постановка задачи такова. Для произвольного набора из $n$ чисел $\mathbf{y}:=\left(y_{0}\right.$, $\left.y_{1}, \ldots, y_{n-1}\right)$ строится интерполяционньй сплайн $s_{r, n}(\mathbf{y}, x) \in S_{r, n}$, удовлетворяющий условиям

$$
s_{r, n}\left(\mathbf{y}, t_{i}\right)=y_{i}, \quad i=0,1, \ldots, n-1 .
$$

Для наборов у вводится $l^{1}$-норма

$$
\|\mathbf{y}\|_{l^{1}}:=\sum_{i=0}^{n-1}\left|y_{i}\right|
$$

и рассматривается норма $L_{r, n}^{*}$ оператора $\mathbf{y} \rightarrow s_{r, n}(\mathbf{y}, x)$ как оператора из $l^{1}$ в $L^{1}[0,1]$.

Основным результатом настояшей работы является следуюшее утверждение, родственное оценке (1.1).

ТЕОРЕма 1. Для норм $L_{r, n}^{*}$ имеет место оценка

$$
L_{r, n}^{*}=\frac{4}{\pi^{2} n} \log \min (r, n)+O\left(\frac{1}{n}\right)
$$

с абсолютной постоянной в остаточном члене.

В п. 3 с помощью формул для интерполяционных сплайнов из работы [3] получено представление норм $L_{r, n}^{*}$, из которого в п. 4 выводится теорема 1 . Второй раздел является вспомогательным. В п. 5 рассмотрена связь величин $L_{r, n}^{*}$ с подобными нормами для непериодических интерполяционных сплайнов, заданных на всей оси.

Как и в [1], на протяжении всей работы будем считать, что $n \geqslant 3$ и $\nu:=[(n-1) / 2]$. Условие $n \geqslant 3$ не ограничивает общность, так как для $n=1$ и $n=2$ оценка (1.3) очевидна.

Все соотношения с $O$-символами в работе являются равномерными относительно всех параметров, но отмечать это мы будем только в формулировках утверждений.

2. Вспомогательные предложения. В представлении интерполяционных сплайнов из [3], которым мы будем пользоваться, и в представлении норм $L_{r, n}^{*}$ участвуют величины

$$
A_{r, j, n}:=\sum_{m=-\infty}^{\infty} \frac{(-1)^{m(r+1)}}{(m n+j)^{r+1}}, \quad B_{r, j, n}:=\sum_{m=-\infty}^{\infty} \frac{(-1)^{m r}}{(m n+j)^{r+1}}
$$

$j=1,2, \ldots, n-1$. Мы придерживаемся обозначений этих величин, которые использовались в [1]. Они отличаются от обозначений, принятых в [3], множителем $(-1)^{r+1}$.

Для величин (2.1) имеют место следующие предложения, первые два из которых были установлены в $[1$, леммы 1 и 2$]$.

ЛЕмма 1. Для всех $j=1, \ldots, n-1$ справедливы оченки

$$
\frac{1}{j^{r+1}}<A_{r, j, n}<\frac{1}{j^{r+1}}+\frac{4}{(n-j)^{r+1}} .
$$


ЛЕмМа 2. При $j=1, \ldots, \nu$ справедливы оценки

$$
0<B_{r, j, n}=\frac{1}{j^{r+1}}-\frac{1}{(n-j)^{r+1}}+O\left(\frac{n-2 j}{n j^{r+1}}\right)
$$

с абсолютной постоянной в остаточном члене.

Заметим, что из (2.3) и левой оценки (2.2) следует, что при $j=1, \ldots, \nu$

$$
\frac{B_{r+1, j, n}}{A_{r, j, n}}=O\left(\frac{1}{j}\right)
$$

ЛЕмма 3. При $j=1,2, \ldots, n-1$ справедливы равенства

$$
A_{r, n-j, n}=A_{r, j, n}, \quad B_{r, n-j, n}=-B_{r, j, n}
$$

ДокАЗАТЕЛьство. Заменим в определении $A_{r, n-j, n}$ индекс суммирования $m$ на $-k-1$. Тогда

$$
A_{r, n-j, n}=\sum_{m=-\infty}^{\infty} \frac{(-1)^{m(r+1)}}{(m n+n-j)^{r+1}}=\sum_{k=-\infty}^{\infty} \frac{(-1)^{(-k-1)(r+1)}}{(-k n-j)^{r+1}}=A_{r, j, n}
$$

Доказательство второго соотношения (2.5) аналогично:

$$
B_{r, n-j, n}=\sum_{m=-\infty}^{\infty} \frac{(-1)^{m r}}{(m n+n-j)^{r+1}}=\sum_{k=-\infty}^{\infty} \frac{(-1)^{(-k-1) r}}{(-k n-j)^{r+1}}=-B_{r, j, n}
$$

Кроме того, будут использоваться следующие оценки из [1, лемма 4].

ЛЕмма 4. Справедливы равномерные относительно всех параметров оценки

$$
\begin{aligned}
& \sum_{j=1}^{\nu} \frac{1}{\nu+1-j}\left[1-\left(\frac{j}{n-j}\right)^{r}\right]=\log \min (r, n)+O(1) \\
& \sum_{j=1}^{\nu} \frac{1+(-1)^{j}}{\nu+1-j}\left[1-\left(\frac{j}{n-j}\right)^{r}\right]=\log \min (r, n)+O(1), \\
& \sum_{j=1}^{\nu} \frac{1}{\nu+1-j}\left[1-\left(\frac{j}{n-j}\right)^{r}\right]\left(\frac{j}{\nu+1}\right)^{r}=O(1) .
\end{aligned}
$$

Нам понадобятся также некоторые свойства величин

$$
\alpha_{j, n}:=\sum_{i=0}^{\nu}(-1)^{i} \cos \frac{j(2 i+1)}{n} \pi, \quad j=1,2, \ldots, n-1 .
$$


Лемма 5. Имеют место следующие соотношения:

$$
\alpha_{n-j, n}=-\alpha_{j, n}
$$

в частности, если п четно и $j=n / 2$, то $\alpha_{j, n}=0 ;$ если $j<n / 2$, то при четном $n$

а при нечетном $n$

$$
\alpha_{j, n}=\frac{1}{2}\left(1+(-1)^{j+\nu}\right) \cos ^{-1} \frac{j}{n} \pi,
$$

$$
\alpha_{j, n}=\frac{1}{2} \cos ^{-1} \frac{j}{n} \pi+O(1)
$$

с абсолютной постоянной в остаточном члене.

ДокАЗАТЕЛЬСтво. Для каждого слагаемого из суммы (2.9) имеем

$$
\cos \frac{(n-j)(2 i+1)}{n} \pi=\cos \left((2 i+1) \pi-\frac{j(2 i+1)}{n} \pi\right)=-\cos \frac{j(2 i+1)}{n} \pi .
$$

Отсюда следует (2.10).

Если $j<n / 2$, то воспользуемся тождеством [4, формула (1.341.6)]

$$
\sum_{i=0}^{\nu}(-1)^{i} \sin (x+i y)=\sin \left(x+\frac{\nu}{2}(y+\pi)\right) \sin \frac{\nu+1}{2}(y+\pi) \cos ^{-1} \frac{y}{2}
$$

при

$$
x=\frac{\pi}{2}+\frac{j}{n} \pi, \quad y=\frac{2 j}{n} \pi .
$$

Тогда получим

$$
\begin{aligned}
\alpha_{j, n} & =\sin \left(\frac{\pi}{2}+\frac{j}{n} \pi+\frac{\nu}{2}\left(\frac{2 j}{n} \pi+\pi\right)\right) \sin \frac{\nu+1}{2}\left(\frac{2 j}{n} \pi+\pi\right) \cos ^{-1} \frac{j}{n} \pi \\
& =\sin ^{2}\left((\nu+1) \frac{j}{n} \pi+\frac{\nu+1}{2} \pi\right) \cos ^{-1} \frac{j}{n} \pi .
\end{aligned}
$$

Если $n$ четно, то $n=2 \nu+2$ и

$$
(\nu+1) \frac{j}{n} \pi+\frac{\nu+1}{2} \pi=(j+\nu+1) \frac{\pi}{2} .
$$

Поэтому

$$
\sin ^{2}\left((\nu+1) \frac{j}{n} \pi+\frac{\nu+1}{2} \pi\right)=\frac{1}{2}\left(1+(-1)^{j+\nu}\right) .
$$

Таким образом, равенство (2.11) доказано.

Пусть теперь $n$ нечетно. Тогда $n=2 \nu+1$ и

$$
(\nu+1) \frac{j}{n} \pi+\frac{\nu+1}{2} \pi=\frac{j \pi}{2 n}+(j+\nu+1) \frac{\pi}{2}=\frac{\pi}{4}-\frac{n-2 j}{4 n} \pi+(j+\nu+1) \frac{\pi}{2} .
$$

Значит,

$$
\sin ^{2}\left((\nu+1) \frac{j}{n} \pi+\frac{\nu+1}{2} \pi\right)=\frac{1}{2}+O\left(\frac{n-2 j}{n}\right) .
$$

Оценка (2.12) вытекает из (2.13) и (2.14), так как

$$
\cos ^{-1} \frac{j}{n} \pi=\sin ^{-1} \frac{n-2 j}{2 n} \pi<\frac{n}{n-2 j} .
$$

Лемма доказана. 


\section{3. Представление норм $L_{r, n}^{*}$.}

ТЕОРема 2. Для норм $L_{r, n}^{*}$ справедливы равномерные по всем параметрам оченки:

$$
L_{r, n}^{*}=\frac{2}{\pi n} \sum_{j=1}^{\nu}\left[1+(-1)^{\nu+j}\right] \frac{B_{r+1, j, n}}{A_{r, j, n}} \operatorname{tg} \frac{j \pi}{n}+O\left(\frac{1}{n}\right)
$$

при четном $n$, а при нечетном $n$

$$
L_{r, n}^{*}=\frac{2}{\pi n} \sum_{j=1}^{\nu} \frac{B_{r+1, j, n}}{A_{r, j, n}} \operatorname{tg} \frac{j \pi}{n}+O\left(\frac{1}{n}\right) .
$$

ДокаЗАТЕльСтво. Пусть $s_{r, n}^{0}(x)$ - фундаментальный сплайн из $S_{r, n}$, решаюший интерполяционную задачу $(1.2)$ для $\mathbf{y}^{0}:=(1,0, \ldots, 0)$. Тогда для произвольного набора $\mathbf{y}=\left(y_{0}, y_{1}, \ldots, y_{n-1}\right)$ имеем

$$
s_{r, n}(\mathbf{y}, x)=\sum_{i=0}^{n-1} y_{i} s_{r, n}^{0}\left(x-t_{i}\right)
$$

Поэтому

$$
\int_{0}^{1}\left|s_{r, n}(\mathbf{y}, x)\right| d x \leqslant \sum_{i=0}^{n-1}\left|y_{i}\right| \int_{0}^{1}\left|s_{r, n}^{0}\left(x-t_{i}\right)\right| d x=\sum_{i=0}^{n-1}\left|y_{i}\right| \int_{0}^{1}\left|s_{r, n}^{0}(x)\right| d x .
$$

Так как для $\mathbf{y}=\mathbf{y}^{0}$ в (3.3) имеет место знак равенства, отсюда следует, что

$$
L_{r, n}^{*}=\int_{0}^{1}\left|s_{r, n}^{0}(x)\right| d x .
$$

Таким образом, задача свелась к изучению интеграла из (3.4).

Так как сплайн $s_{r, n}^{0}(x)$ имеет период 1 , то

$$
L_{r, n}^{*}=\int_{t_{0}}^{1+t_{0}}\left|s_{r, n}^{0}(x)\right| d x=\sum_{i=0}^{n-1} \int_{t_{i}}^{t_{i+1}}\left|s_{r, n}^{0}(x)\right| d x .
$$

Из определения $s_{r, n}^{0}(x)$ в силу единственности интерполяционных сплайнов следует равенство

$$
s_{r, n}^{0}\left(t_{0}+x\right)=s_{r, n}^{0}\left(t_{0}-x\right) .
$$

А. А. Женсыкбаев [3, теорема 1] установил, что если $n$ четно, то при $i=0,1, \ldots, \nu$

$$
\operatorname{sign} s_{r, n}^{0}(x)=(-1)^{i}, \quad x \in\left(t_{i}, t_{i+1}\right),
$$

а если $n$ нечетно, равенство (3.6) имеет место при всех $i=0,1, \ldots, n-1$. Поэтому, учитывая (3.5), имеем

$$
L_{r, n}^{*}=2 \sum_{i=0}^{\nu}(-1)^{i} \int_{t_{i}}^{t_{i+1}} s_{r, n}^{0}(x) d x-\frac{1-(-1)^{n}}{2}(-1)^{\nu} \int_{t_{\nu}}^{t_{\nu+1}} s_{r, n}^{0}(x) d x .
$$


Для сплайнов $s_{r, n}^{0}(x)$ справедливо представление [3, формула (7)]

$$
s_{r, n}^{0}(x)=\frac{1}{n}+\frac{(-1)^{r+1}}{n} \sum_{j=1}^{n-1} A_{r, j, n}^{-1} \sum_{m=-\infty}^{\infty} \frac{\cos 2 \pi\left[(m n-j) x+j t_{0}\right]}{(m n-j)^{r+1}} .
$$

Пользуясь этим представлением и равенством

$$
\begin{aligned}
\int_{t_{i}}^{t_{i+1} \cos 2 \pi\left[(m n-j) x+j t_{0}\right] d x=} & \frac{1}{2 \pi(m n-j)} 2 \sin \pi \frac{m n-j}{n} \\
& \times \cos 2 \pi\left[(m n-j)\left(t_{0}+\frac{2 i+1}{2 n} \pi\right)+j t_{0}\right] \\
= & \frac{1}{\pi(m n-j)}(-1)^{m(r+1)+1} \sin \frac{j \pi}{n} \cdot \cos \frac{j(2 i+1)}{n} \pi
\end{aligned}
$$

получаем

$$
\begin{aligned}
\int_{t_{i}}^{t_{i+1}} s_{r, n}^{0}(x) d x= & \frac{1}{n^{2}}+\frac{(-1)^{r+1}}{\pi n} \\
& \times \sum_{j=1}^{n-1} A_{r, j, n}^{-1} \sin \frac{j \pi}{n} \cos \frac{j(2 i+1)}{n} \pi \sum_{m=-\infty}^{\infty} \frac{(-1)^{m(r+1)+1}}{(m n-j)^{r+2}} \\
= & \frac{1}{n^{2}}+\frac{1}{\pi n} \sum_{j=1}^{n-1} \sin \frac{j \pi}{n} \cos \frac{j(2 i+1)}{n} \pi \frac{B_{r+1, j, n}}{A_{r, j, n}}
\end{aligned}
$$

Заметим, что при $n$ четном последнее слагаемое в правой части (3.7) равно нулю.

Если же $n$ нечетно, то согласно (3.8) модуль последнего слагаемого из правой части (3.7) не превосходит суммы

$$
\frac{1}{n^{2}}+\frac{1}{n} \sum_{j=1}^{n-1} \sin \frac{j \pi}{n} \frac{\left|B_{r+1, j, n}\right|}{A_{r, j, n}}=\frac{1}{n^{2}}+\frac{2}{n} \sum_{j=1}^{\nu} \sin \frac{j \pi}{n} \frac{B_{r+1, j, n}}{A_{r, j, n}}
$$

здесь мы воспользовались равенствами (2.5).

Полученное выражение в силу (2.4) есть

$$
\frac{1}{n^{2}}+O\left(\frac{1}{n} \sum_{j=1}^{\nu} \frac{j}{n} \frac{1}{j}\right)=O\left(\frac{1}{n}\right)
$$

Поэтому из (3.7) и (3.8) следует, что

$$
L_{r, n}^{*}=\frac{2}{\pi n} \sum_{j=1}^{n-1} \sin \frac{j \pi}{n} \frac{B_{r+1, j, n}}{A_{r, j, n}} \sum_{i=0}^{\nu}(-1)^{i} \cos \frac{j(2 i+1)}{n} \pi+O\left(\frac{1}{n}\right) .
$$

Пользуясь обозначением (2.9), эту оценку можно записать так:

$$
L_{r, n}^{*}=\frac{2}{\pi n} \sum_{j=1}^{n-1} \sin \frac{j \pi}{n} \frac{B_{r+1, j, n}}{A_{r, j, n}} \alpha_{j, n}+O\left(\frac{1}{n}\right) .
$$


Если $n$ четно и $j=n / 2=\nu+1$, то согласно лемме $5 \alpha_{\nu+1, n}=0$. Поэтому для всех $n$ имеем

$$
\begin{aligned}
\sum_{j=1}^{n-1} \sin \frac{j \pi}{n} \frac{B_{r+1, j, n}}{A_{r, j, n}} \alpha_{j, n}= & \sum_{j=1}^{\nu} \sin \frac{j \pi}{n} \frac{B_{r+1, j, n}}{A_{r, j, n}} \alpha_{j, n} \\
& +\sum_{j=1}^{\nu} \sin \frac{(n-j) \pi}{n} \frac{B_{r+1, n-j, n}}{A_{r, n-j, n}} \alpha_{n-j, n} .
\end{aligned}
$$

Таким образом, из (3.9) в силу равенств (2.5) и (2.10) получаем

$$
L_{r, n}^{*}=\frac{4}{\pi n} \sum_{j=1}^{\nu} \sin \frac{j \pi}{n} \frac{B_{r+1, j, n}}{A_{r, j, n}} \alpha_{j, n}+O\left(\frac{1}{n}\right) .
$$

Если $n$ четно, то оценка (3.1) сразу вытекает из (3.10) и (2.11). А если $n$ нечетно, то чтобы из (3.10) и (2.12) получить (3.2), нужно воспользоваться тем, что в силу (2.4)

$$
\sum_{j=1}^{\nu} \sin \frac{j \pi}{n} \frac{B_{r+1, j, n}}{A_{r, j, n}}=O\left(\sum_{j=1}^{\nu} \frac{j}{n} \frac{1}{j}\right)=O(1) .
$$

Теорема доказана.

4. Доказательство теоремы 1. Будем исходить из оценок теоремы 2. Рассмоторим сначала нечетные $n$. Положим

$$
P_{r, n}:=\sum_{j=1}^{\nu} \frac{B_{r+1, j, n}}{A_{r, j, n}} \operatorname{tg} \frac{j \pi}{n} .
$$

Пусть $\mu:=[\nu / 2]$. Тогда $\mu \leqslant \nu / 2<n / 4$ и, значит, для $j \leqslant \mu$ имеем

$$
\operatorname{tg} \frac{j \pi}{n}=O\left(\frac{j}{n}\right)
$$

Поэтому согласно (2.4)

$$
\sum_{j=1}^{\mu} \frac{B_{r+1, j, n}}{A_{r, j, n}} \operatorname{tg} \frac{j \pi}{N}=O\left(\sum_{j=1}^{\mu} \frac{1}{j} \frac{j}{n}\right)=O(1) .
$$

Таким образом,

$$
P_{r, n}:=\sum_{j=\mu+1}^{\nu} \frac{B_{r+1, j, n}}{A_{r, j, n}} \operatorname{tg} \frac{j \pi}{n}+O(1) .
$$

Так как для $j \leqslant \nu<n / 2$ справедлива оценка

$$
\operatorname{tg} \frac{j \pi}{n}=\operatorname{ctg}\left(\frac{\pi}{2} \frac{n-2 j}{n}\right)=\frac{2}{\pi} \frac{n}{n-2 j}+O(1)=\frac{4}{\pi} \frac{j}{n-2 j}+O(1),
$$

TO

$$
P_{r, n}=\frac{4}{\pi} \sum_{j=\mu+1}^{\nu} \frac{j}{n-2 j} \frac{B_{r+1, j, n}}{A_{r, j, n}}+O(1)
$$


поскольку в силу (2.4)

$$
\sum_{j=\mu+1}^{\nu} \frac{B_{r+1, j, n}}{A_{r, j, n}}=O\left(\sum_{j=\mu+1}^{\nu} \frac{1}{j}\right)=O(1) .
$$

Подставим теперь в (4.1) вместо величин $B_{r+1, j, n}$ их выражения по формуле (2.3). Так как в силу левой оценки (2.2)

$$
\sum_{j=\mu+1}^{\nu} \frac{j}{n-2 j} \frac{1}{A_{r, j, n}} \frac{n-2 j}{n j^{r+2}}<\sum_{j=\mu+1}^{\nu} \frac{1}{n}<1,
$$

получим

$$
P_{r, n}=\frac{4}{\pi} \sum_{j=\mu+1}^{\nu} \frac{j}{n-2 j} \frac{1}{A_{r, j, n}}\left(\frac{1}{j^{r+2}}-\frac{1}{(n-j)^{r+2}}\right)+O(1) .
$$

Поскольку для нечетных $n$, которые мы сейчас рассматриваем, $n-2 j=2 \nu+1-2 j$, то

$$
\frac{1}{n-2 j}=\frac{1}{2(\nu+1-j)}+O\left(\frac{1}{(\nu+1-j)^{2}}\right) \text {. }
$$

Поэтому с помощью левой оценки (2.2) находим

$$
P_{r, n}=\frac{2}{\pi} \sum_{j=\mu+1}^{\nu} \frac{j}{\nu+1-j} \frac{1}{A_{r, j, n}}\left(\frac{1}{j^{r+2}}-\frac{1}{(n-j)^{r+2}}\right)+O(1) .
$$

Из оценок (2.2) следует, что

$$
\frac{1}{A_{r, j, n}}=j^{r+1}+O\left(\frac{j^{2(r+1)}}{(n-j)^{r+1}}\right) .
$$

Прежде чем подставить это выражение в (4.3), заметим, что сумма

$$
\begin{aligned}
\sum_{j=\mu+1}^{\nu} & \frac{j}{\nu+1-j}\left(\frac{1}{j^{r+2}}-\frac{1}{(n-j)^{r+2}}\right) \frac{j^{2(r+1)}}{(n-j)^{r+1}} \\
& =\sum_{j=\mu+1}^{\nu} \frac{1}{\nu+1-j}\left(1-\left(\frac{j}{n-j}\right)^{r+2}\right)\left(\frac{j}{n-j}\right)^{r+1}
\end{aligned}
$$

равномерно ограничена в силу (2.8).

Таким образом, из (4.3) и (4.4) следует, что

$$
\begin{aligned}
P_{r, n} & =\frac{2}{\pi} \sum_{j=\mu+1}^{\nu} \frac{j}{\nu+1-j} j^{r+1}\left(\frac{1}{j^{r+2}}-\frac{1}{(n-j)^{r+2}}\right)+O(1) \\
& =\frac{2}{\pi} \sum_{j=\mu+1}^{\nu} \frac{1}{\nu+1-j}\left(1-\left(\frac{j}{n-j}\right)^{r+2}\right)+O(1) .
\end{aligned}
$$

Так как при $j \leqslant \mu$ имеем $\nu+1-j \geqslant \nu+1-\mu>\mu$, то (4.5) равносильно оценке

$$
P_{r, n}=\frac{2}{\pi} \sum_{j=1}^{\nu} \frac{1}{\nu+1-j}\left(1-\left(\frac{j}{n-j}\right)^{r+2}\right)+O(1) .
$$


Оценка (1.3) для нечетных $n$ вытекает из (4.6) и (2.6).

Если $n$ четно, то, пользуясь вместо (4.2) равенством $n-2 j=2(\nu+1-j)$, точно так же, как была доказана оценка (4.6), приходим к оценке

$$
\begin{aligned}
\sum_{j=1}^{\nu}[1 & \left.+(-1)^{\nu+j}\right] \frac{B_{r+1, j, n}}{A_{r, j, n}} \operatorname{tg} \frac{j \pi}{n}+O\left(\frac{1}{n}\right) \\
& =\frac{2}{\pi} \sum_{j=1}^{\nu} \frac{1+(-1)^{\nu+j}}{\nu+1-j}\left(1-\left(\frac{j}{n-j}\right)^{r+2}\right)+O(1) .
\end{aligned}
$$

Оценка (1.3) для четных $n$ следует из (4.7) и (2.7).

Теорема 1 доказана.

5. Сравнение с непериодическим случаем. Задача об асимптотическом поведении норм $L_{r, n}^{*}$ близка к следующей задаче, рассматривавшейся в работе [5].

Пусть $S_{r}-$ множество сплайнов степени $r$ дефекта 1 с узлами в целых точках $i,-\infty<$ $i<+\infty$. Для последовательностей чисел $\mathbf{z}:=\left(z_{i},-\infty<i<+\infty\right)$, удовлетворяющих условию

$$
\|\mathbf{z}\|_{l^{1}}:=\sum_{i=-\infty}^{\infty}\left|z_{i}\right|<\infty
$$

строятся интерполяционные сплайны $\sigma_{r}(\mathbf{z}, x) \in S_{r}$ такие, что

$$
\sigma_{r}\left(\mathbf{z}, \tau_{i}\right)=z_{i}, \quad-\infty<i<+\infty
$$

где

$$
\tau_{i}:=i+\frac{1+(-1)^{r}}{4} .
$$

Спрашивается, как ведут себя нормы $\mathcal{L}_{r}$ оператора $\mathbf{z} \rightarrow \sigma_{r}(\mathbf{z}, x)$ как оператора из $l^{1}$ в $L^{1}(-\infty,+\infty)$.

В $[5$, теорема 2.1$]$ для величин $\mathcal{L}_{r}$ получена оценка

$$
\mathcal{L}_{r}=\frac{4}{\pi^{2}} \log r+\frac{2 A}{\pi}+\frac{4}{\pi^{2}}\left(\log \frac{4}{\pi}+\gamma\right)+o(1), \quad r \rightarrow \infty,
$$

где

$$
A:=\int_{0}^{\pi}\left(\frac{1}{t} \operatorname{tg} \frac{t}{2}-\frac{2}{\pi(\pi-t)}\right) d t
$$

и $\gamma$ - постоянная Эйлера.

Пусть $\sigma_{r}^{0}(x)$ - фундаментальный сплайн из $S_{r}$, решаюший интерполящионную задачу $(5.2)$ для последовательности $\mathbf{z}^{0}:=\left(z_{0}=1, z_{i}=0\right.$ при $\left.i \neq 0\right)$.

Известно, что для каждого $r=1,2, \ldots$ сплайн $\sigma_{r}^{0}(x)$ принадлежит пространству $L^{1}(-\infty,+\infty)$, см., например, [6, теорема 12]. Этот факт очевидным образом вытекает из результатов работы [7], но в явном виде в [7] он не был отмечен.

При доказательстве оценки (5.3) в [5] было установлено равенство

$$
\mathcal{L}_{r}=\int_{-\infty}^{\infty}\left|\sigma_{r}^{0}(x)\right| d x
$$


Чтобы сравнить оценки (5.3) и (1.3), преобразуем интеграл из (5.4) следующим образом:

$$
\mathcal{L}_{r}=\sum_{k=-\infty}^{\infty} \int_{0}^{n}\left|\sigma_{r}^{0}(x+k n)\right| d x=\int_{0}^{n} \sum_{k=-\infty}^{\infty}\left|\sigma_{r}^{0}(x+k n)\right| d x .
$$

Последний шаг обосновывается ссылкой на теорему Б. Леви о предельном переходе под знаком интеграла (теорему Лебега о монотонной сходимости).

Функция

$$
\sigma_{r, n}^{*}(x):=\sum_{k=-\infty}^{\infty} \sigma_{r}^{0}(x+k n)
$$

представляет собой сплайн периода $n$ с узлами в целых точках.

Понятно, что сплайн $\sigma_{r, n}^{*}(x)$ связан со сплайном $s_{r, n}^{0}(x)$, для которого справедливо равенство (3.4), соотношением

Поэтому

$$
\sigma_{r, n}^{*}(x)=s_{r, n}^{0}\left(\frac{x}{n}\right)
$$

$$
\int_{0}^{n}\left|\sigma_{r, n}^{*}(x)\right| d x=n \int_{0}^{1}\left|s_{r, n}^{0}(x)\right| d x=n L_{r, n}^{*} .
$$

В силу (5.3) и (1.3) равномерно относительно параметров $r$ и $n$

$$
\mathcal{L}_{r}=\int_{0}^{n} \sum_{k=-\infty}^{\infty}\left|\sigma_{r}^{0}(x+k n)\right| d x=\frac{4}{\pi^{2}} \log r+O(1)
$$

и

$$
n L_{r, n}^{*}=\int_{0}^{n}\left|\sum_{k=-\infty}^{\infty} \sigma_{r}^{0}(x+k n)\right| d x=\frac{4}{\pi^{2}} \log \min (r, n)+O(1) .
$$

Таким образом, здесь имеет место тот же эффект, что и отмеченный в [1] эффект для интерполяционных сплайнов в равномерной метрике, когда $\log r$ в главном члене асимптотики констант Лебега для произвольных сплайнов заменяется на $\log \min (r, n)$ при переходе к сплайнам периода $n$.

\section{СПИСОК ЦИТИРОВАННОЙ ЛИТЕРАТУРЫ}

[1] Субботин Ю. Н., Теляковский С. А. Асимптотика констант Лебега периодических интерполяционных сплайнов с равноотстоящими узлами // Матем. сб. 2000. Т. 191. №8. C. $131-140$.

[2] Стечкин С. Б., Субботин Ю. Н. Сплайны в вычислительной математике. М.: Физматгиз, 1976.

[3] ЖКенсыкбаев А. А. Точные оценки равномерного приближения непрерывных периодических функций сплайнами $r$-го порядка // Матем. заметки. 1973. Т. 13. № 2. С. 217-228.

[4] Градштейн И. С., Рыжик И. М. Таблицы интегралов, сумм, рядов и произведений. М.: Физматгиз, 1962.

[5] Marsden M. J., Richards F. B., Riemenschneider S. D. Cardinal splineinterpolation operators on $l^{p}$ data // Indiana University Math. J. 1975. V. 24. P. 677-689.

[6] Schoenberg I. J. Cardinal interpolation and spline functions // J. Approx. Theory. 1969. V. 2. P. $167-206$.

[7] Субботин Ю. Н. О связи между конечными разностями и соответствующими производными // Тр. МИАН. 1965. Т. 78. С. 24-42.

Институт математики и механики Уральского отделения РАН,

г. Екатеринбург 